\title{
Influência da variação do tamanho de grão austenítico na qualidade superficial no processo de microfresamento do aço AISI H13 endurecido
}

\author{
Influence of the austenite grain size variation \\ on the surface finishing in the micromilling \\ process of the hardened AISI H13steel
}

\begin{abstract}
${ }^{1}$ Departamento de Engenharia Mecânica, Universidade de Aveiro, Campus Universitário de Santiago, 3810 193, Aveiro, Portugal, e-mail: carlos.lauro@ua.pt

${ }^{2}$ Departamento de Engenharia Mecânica, Universidade Federal de São João del-Rei, Praça Frei Orlando 170, Centro, São João del-Rei, Minas Gerais, CEP: 360307-352, e-mail: sergiolmrf@gmail.com

${ }^{3}$ Departamento de Engenharia Civil, Universidade Federal de São Carlos, Rodovia Washington Luis, km 235, CEP: 13.565-905 - São Carlos - São Paulo, e-mail: alchristoforo@ yahoo.com.br

${ }^{4}$ Departamento de Engenharia Mecânica, Universidade Federal de São João del-Rei, Praça Frei Orlando 170, Centro, São João del-Rei, Minas Gerais, CEP: 360307-352, e-mail: lincoln@ufsj.edu.br
\end{abstract}

\begin{abstract}
RESUMO
O foco deste trabalho foi o estudo da influência do tamanho de grão austenítico na qualidade superficial durante o processo de micro fresamento de aços endurecidos. Corpos de prova com durezas de $46 \mathrm{HRC}$ e com diferentes tamanhos de grão foram fresados com micro fresas de topo com $0,5 \mathrm{~mm}$ de diâmetro. Nos testes experimentais foram empregadas duas velocidades de corte e dois avanços por dente. Os resultados foram analisados empregando-se uma análise de variância e demonstraram que a velocidade de corte e o avanço não tiveram influência no comportamento da rugosidade considerando os parâmetros Ra e Rz. Dessa forma, pode-se observar que o fator mais importante foi o tamanho de grão que influenciou significativamente os valores de rugosidade Ra e Rz. Além disso, observou-se também que a interação das variáveis de entrada não demonstrou influência significativa sobre as respostas rugosidade Ra e Rz.
\end{abstract}

Palavras-chave: microfresamento, tamanho de grão, aços endurecidos, rugosidade Ra e Rz.

\section{ABSTRACT}

The focus of this work was to study the influence of austenite grain size in the surface finish during micro milling of hardened steels. Work pieces with hardness of $46 \mathrm{HRC}$ and different grain sizes were milled with micro end mills with a diameter of $0.5 \mathrm{~mm}$. The experimental tests were carried out with two cutting speeds and two feed rates. The results were evaluated using an analysis of variance, and they demonstrated that the cutting speed and the feed rate have no influence on the responses surface roughness $\mathrm{Ra}$ and $\mathrm{Rz}$. Thus, it could be observed that the most important factor was the grain size that influenced the roughness $\mathrm{Ra}$ and $\mathrm{Rz}$, significantly. Moreover, it was also observed that the interaction of input parameters did not show significant influence on the response surface roughness $\mathrm{Ra}$ and $\mathrm{Rz}$.

Keywords: micromilling, grain size, hardened steels, Surface Roughness Ra and Rz. 


\section{INTRODUÇÃO}

$\mathrm{O}$ processo de microfresamento apresenta grande destaque entre todos os processos de microusinagem. Isso ocorre pelo fato de esse processo apresentar ótima precisão e baixa rugosidade, porém ele apresenta baixa taxa de remoção de material. Em grande parte, o processo de microfresamento está relacionado com a fabricação de pequenos detalhes em moldes e matrizes e a usinagem de pequenos componentes. Devido ao fato de ainda apresentar uma modesta aplicação no cenário industrial, a microusinagem de aços ferramenta tem vários desafios, como o fenômeno imprevisível relacionado ao desgaste e vida das ferramentas, o estudo das forças de corte e o conhecimento preciso da formação do cavaco em microescala [1]. De acordo com [2], o processo de microfresamento pode ser usado na indústria para a fabricação de micropartes devido à grande flexibilidade do processo e das máquinas-ferramenta. Essa flexibilidade inclui o diversificado tamanho dos componentes, a forma destes, além da possibilidade dos equipamentos em produzirem uma variedade de geometrias.

Entretanto, o desempenho de microcomponentes e microssistemas está relacionado diretamente com a qualidade da superfície usinada. Para se ter sucesso no processo de microinjeção, alguns autores sugerem que os moldes devem ter rugosidade inferior ou igual a $1 \mu \mathrm{m}$. Isso se deve ao fato da exigência de minimizar os escoamentos turbulentos durante o processo de injeção [3]. De acordo com [4], o efeito da espessura mínima do cavaco tem forte influência sobre a superfície usinada. Não muito diferente do processo de macrousinagem, em algumas condições de usinagem, os efeitos do avanço podem ser significativos na variação da textura dos componentes usinados.

Diversos estudos têm sido conduzidos para identificar a influência da rugosidade das ferramentas e coberturas devido ao efeito de escala nos processos de microusinagem, pois o efeito da rugosidade da ferramenta afeta o desgaste das ferramentas e influencia a degradação da superfície usinada [5] . Em alguns casos, os efeitos do fluido lubrirrefrigerante têm influência significativa no desgaste de ferramentas e na rugosidade de superfícies usinadas. Alguns autores [] investigaram o microfresamento da liga NAK80 com fresas de $2 \mathrm{~mm}$ de diâmetro e observaram que o aumento da rugosidade Ra foi significativo com a aplicação de fluido lubrirrefrigerante.

Normalmente, os materiais de engenharia apresentam as mesmas características físicas quando são utilizados para a fabricação de micro e macrocomponentes. Dessa forma, os materiais de engenharia são, em geral, constituídos de milhões de pequenos cristais. O conjunto de átomos constituintes, de um material, empilhados em um padrão regular e repetitivo, é denominado cristalino. E um cristalino individual em uma microestrutura policristalina é chamado de grão [7]. Quando esse material é submetido a uma temperatura elevada, acima do limite superior da zona crítica, por um determinado tempo, o carbono e os elementos de liga passam por uma transformação, iniciada nos contornos dos grãos e nos grãos menores, dissolvendo-se na austenita. Os produtos de transformação da austenita, denominados ferrita e perlita, iniciam-se nos contornos de grão por nucleação e crescimento. Em temperatura e tempo maiores, os grãos passam por alteração do tamanho médio. Esse aumento pode melhorar a temperabilidade do material, mas pode deteriorar as propriedades mecânicas do material $[7, \underline{8}]$.

De acordo com [9], o tamanho de grão austenítico que é atingido antes que o metal seja resfriado novamente à temperatura ambiente é importante na determinação das propriedades físicas da estrutura final, incluindo a resposta ao endurecimento do aço. Portanto, a variação de grão austenítico em um aço pode influenciar significativamente suas propriedades incluindo as propriedades relacionadas com os processos de usinagem com cunha definida. O tamanho de grão austenítico para aços de aplicação mecânica situa-se em uma escala de 1 a 9 de acordo com o padrão ASTM que corresponde ao número de grãos por polegada quadrada. Assim, um aço com grãos finos de tamanho de grão austenítico ASTM 7, por exemplo, tem quatro vezes mais grãos que um aço de tamanho de grão austenítico ASTM 3.

Dessa forma, a variação do tamanho de grão austenítico pode ter grande influência no efeito de escala considerando o processo de microusinagem. Nos processos de macrousinagem, a relação do tamanho da ferramenta com os grãos que compõem o material pode ser considerada desprezível, pois em uma operação de fresamento podem ser removidos vários grãos simultaneamente, não havendo, então, influência do tamanho grão no processo. Entretanto, quando são fabricados microcomponentes, onde rasgos e furos apresentam dimensões menores que $1 \mathrm{~mm}$, podendo em alguns casos apresentar dimensões de $0,1 \mathrm{~mm}$, a relação do tamanho de grão pode ser significante.

Considerando os aspectos de qualidade superficial, geralmente a rugosidade de um determinado componente está relacionada com a variação dos parâmetros de usinagem. No processo de macrofresamento, o avanço tem forte influência no acabamento de uma determinada geometria. Avaliando o processo de microfresamento, pode-se definir que as marcas de corte devido ao batimento da ferramenta influenciam na rugosidade. Em alguns casos, essas marcas podem acontecer duas vezes mais indicando que uma aresta de corte pode atuar mais que a outra, porém mais estudos precisam ser desenvolvidos para comprovar essa condição efetivamente [10]. 
A rugosidade de uma determinada região usinada corresponde a uma ondulação da superfície medida em escala micrométrica. A rugosidade Ra corresponde a um parâmetro de amplitude muito usada na indústria que corresponde ao desvio aritmético médio em relação a uma linha média. Diversos parâmetros influenciam a rugosidade Ra, tais como; a rigidez da máquina ferramenta, os parâmetros de corte, o material usinado e a estratégia de corte usada [11]. Estudos experimentais mostram que a rugosidade cresce com o aumento do avanço por dente para processos de macrousinagem. Além disso, a condição de afiação da ferramenta de corte e o raio de arredondamento da ferramenta são fatores efetivos para a variação da rugosidade. Considerando os trabalhos de macrousinagem, pode-se afirmar que o desgaste das ferramentas também aumenta a rugosidade das superfícies usinadas [12].

Dessa forma, este trabalho foi desenvolvido para analisar a influência do tamanho de grão austenítico na qualidade superficial durante o microfresamento de aços endurecidos com diferentes tamanhos de grão. Assim, buscou-se compreender qual foi a relação de escala entre as dimensões da ferramenta de corte e a rugosidade gerada após o fresamento de microcanais.

\section{MATERIAIS E MÉTODOS}

Os testes de microusinagem foram realizados em um centro de usinagem vertical Discovery 560 da Romi, do Laboratório de Usinagem da Universidade Federal de São João del-Rei, com curso da mesa de $560 \mathrm{~mm}$ no eixo longitudinal "X", $406 \mathrm{~mm}$ no eixo latitudinal "Y" e $508 \mathrm{~mm}$ no eixo vertical "Z". A potência máxima do Centro de Usinagem era de $15 \mathrm{~kW}$ e a rotação máxima de $10.000 \mathrm{rpm}$, equipado com comando numérico Siemens modelo SINUMERIC 810D. Para que fossem obtidas maiores velocidades de corte neste experimento, não foi utilizada a rotação do centro vertical de usinagem, mas foi acoplado ao eixo-árvore do centro vertical de usinagem um cabeçote de ultraprecisão e alta rotação da Nakanishi modelo HES510, com rotação máxima de $50.000 \mathrm{rpm}$ e potência no eixo de $300 \mathrm{~W}$.

Nos testes de microusinagem, foram utilizadas fresas inteiriças de metal duro de diâmetro 0,5 mm com duas arestas cortantes, fornecidas pela SANDVIK Coromant, código R216.32-00530-AE05G 1620. Para o desenvolvimento deste trabalho, foram confeccionados corpos de prova com $11 \mathrm{~mm}$ de aresta em aço AISI H13, com composição $0,40 \%$ de C; $1,00 \% \mathrm{Si} ; 0,35 \%$ de $\mathrm{Mn} ; 5,20 \%$ de $\mathrm{Cr} ; 1,50 \%$ de Mo; e $0,90 \%$ de V, fornecido pela Villares Metal. Os corpos de prova receberam tratamento térmico para atingir a dureza de trabalho em torno de $46^{ \pm 2} \mathrm{HRC}$, a fim de se obterem diferentes tamanhos de grãos austeníticos.

A variação do tamanho do grão austenítico teve como objetivo avaliar os efeitos do corte considerando a escala de microusinagem em relação ao contorno do grão. Para a obtenção dos diferentes tamanhos de grão austeníticos, os corpos de prova foram tratados termicamente em um forno de atmosfera controlada onde permaneceram por tempos determinados, sendo resfriados em óleo para têmpera à temperatura ambiente de $25^{\circ} \mathrm{C}^{ \pm 3}$ [7] conforme Tabela 1. Assim, obtiveram-se os tamanhos médios de grãos austeníticos de 39,9 $\mu \mathrm{m}$, Figura 1, e $497 \mu \mathrm{m}$, Figura 2.

Tabela 1: Parâmetros para Crescimento de Grão Austenítico

\begin{tabular}{ccccccc}
\hline Condição & $\begin{array}{c}\text { Tamanho de } \\
\text { Grão }(\mu \mathrm{m})\end{array}$ & $\begin{array}{c}\text { Tamanho } \\
\text { de Grão } \\
(\text { ASTM })\end{array}$ & $\begin{array}{c}\text { Dureza } \\
\text { HRC }\end{array}$ & $\begin{array}{c}\text { Temperatura } \\
\left({ }^{\circ} \mathrm{C}\right)\end{array}$ & $\begin{array}{c}\text { Tempo } \\
(\text { min. })\end{array}$ & $\begin{array}{c}\text { Meio de } \\
\text { Resfriamento }\end{array}$ \\
\hline H1 & 497 & 0 & 44 & 1250 & 120 & Óleo Têmpera \\
H2 & 39,9 & 6 & 46 & 1025 & 45 & 100 \\
\hline
\end{tabular}
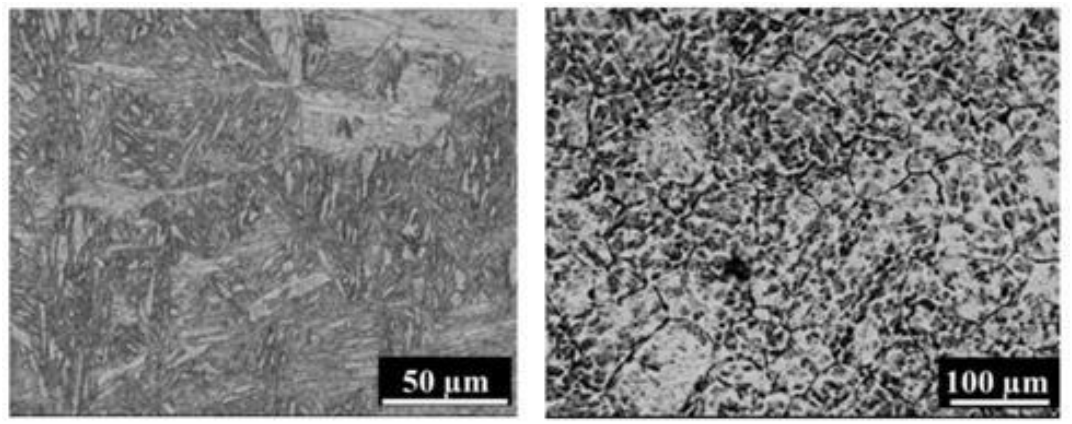

Figura 1: Análise Metalográfica do aço AISI H13 com tamanho de grão de 39,9 $\mu \mathrm{m}$ 

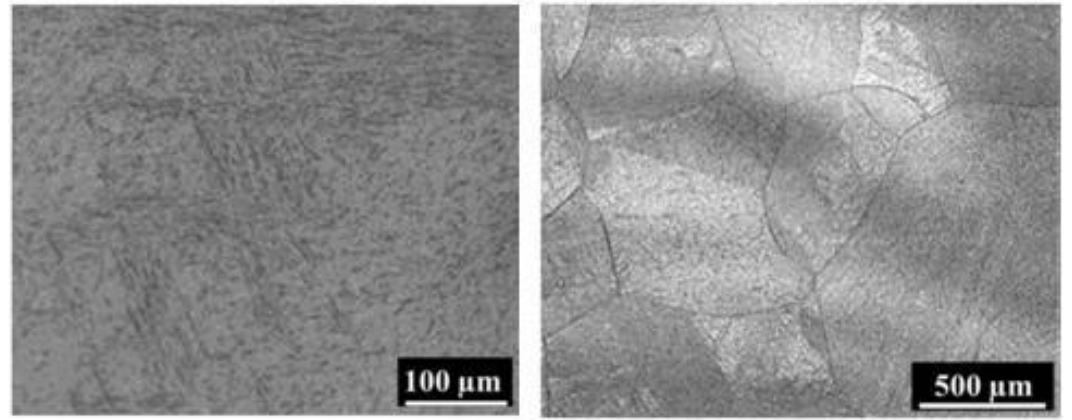

Figura 2: Análise Metalográfica do aço AISI H13 com tamanho de grão de $497 \mu \mathrm{m}$

Os parâmetros de entrada utilizados no experimento, como velocidade de corte $\left(\mathrm{v}_{\mathrm{c}}\right)$, avanço por dente $\left(\mathrm{f}_{\mathrm{Z}}\right)$, profundidade de corte $\left(\mathrm{a}_{\mathrm{p}}\right)$ e profundidade radial de corte $\left(\mathrm{a}_{\mathrm{e}}\right)$, foram selecionados mediante informações do fabricante da ferramenta. As condições descritas na Tabela 2 foram aplicadas nos dois diferentes tamanhos de grão austenítico. Portanto, um planejamento fatorial completo do tipo $2^{\mathrm{k}}$ com três réplicas resultou em um total de 24 experimentos. A Tabela 2 mostra os valores dos parâmetros de entrada utilizados nos experimentos de microusinagem. Dessa forma, foram produzidos entalhes em todo a extensão do corpo de prova com 0,5 mm de largura (mesmo diâmetro da ferramenta) e profundidade de corte $\left(\mathrm{a}_{\mathrm{p}}\right)$ de $0,025 \mathrm{~mm}$.

Tabela 2: Valores dos Parâmetros de Corte para os Ensaios de Microfresamento

\begin{tabular}{ccccc}
\hline Condição & $\begin{array}{c}\text { Profundidade axial } \\
\text { de corte }\left(\mathrm{a}_{\mathrm{p}}\right) \\
(\mathrm{mm})\end{array}$ & $\begin{array}{c}\text { Profundidade radial } \\
\text { de corte }\left(\mathrm{a}_{\mathrm{e}}\right) \\
(\mathrm{mm})\end{array}$ & $\begin{array}{c}\text { Velocidade } \\
\text { de corte }(\mathrm{vc}) \\
(\mathrm{m} / \mathrm{min})\end{array}$ & $\begin{array}{c}\text { Avanço por } \\
\text { dente }\left(\mathrm{f}_{\mathrm{Z}}\right) \\
(\mathrm{mm} / \mathrm{rot})\end{array}$ \\
\hline 1 & 0,025 & 0,5 & 33 & 0,001 \\
2 & 0,025 & 0,5 & 33 & 0,005 \\
3 & 0,025 & 0,5 & 77 & 0,001 \\
4 & 0,025 & 0,5 & 77 & 0,005 \\
\hline
\end{tabular}

Para análise da rugosidade gerada pelo corte, foi utilizado um Rugosímetro SJ-400 da Mitutoyo ${ }^{\mathrm{TM}}$, sendo a rugosidade dos corpos de prova medida no sentido de avanço, conforme pode ser observado na Figura 3. A medição foi realizada por toda a extremidade dos entalhes. $O$ parâmetro de medição foi o percurso de amostragem de $2 \mathrm{~mm}$ para rugosidade abaixo de $0,1 \mu \mathrm{m}$ e para rugosidade entre 0,1 e $2,0 \mu \mathrm{m}$.

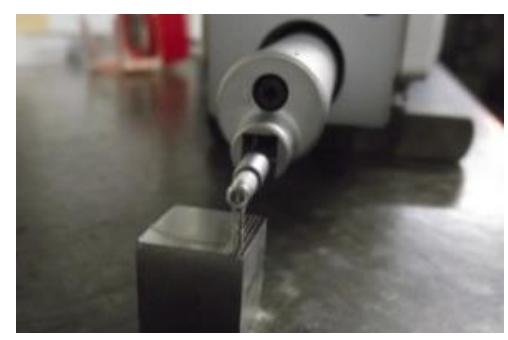

Figura 3: Medição da rugosidade

\section{ANÁLISE DOS RESULTADOS}

Após a medição dos valores de rugosidade, foram calculadas as médias para cada experimento conforme os valores apresentados na Tabela 3. Para melhor entendimento, aplicou-se a Análise de Variância, ANOVA, onde se observou, para a rugosidade Ra, Tabela 4, que o fator mais significante foi o tamanho do grão, inversamente proporcional, Figuras 4 e 5. Pode-se observar na Tabela 3 que o máximo valor de rugosidade

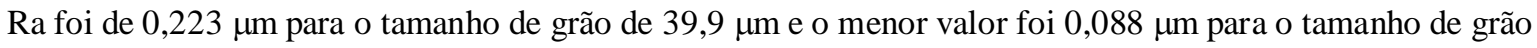
de $497 \mu \mathrm{m}$. 
Tabela 3: Valores médios de rugosidade obtidos no ensaio experimental

\begin{tabular}{cccccc}
\hline \multirow{2}{*}{$\begin{array}{c}\text { Velocidade } \\
\text { de corte } \\
(\mathrm{m} / \mathrm{min})\end{array}$} & $\begin{array}{c}\text { Avanço } \\
\text { por dente } \\
(\mu \mathrm{m} / \mathrm{rot})\end{array}$ & \multicolumn{4}{c}{ Tamanho de grão $[\mu \mathrm{m}]$} \\
\cline { 4 - 7 } & 1 & 0,145 & 0,142 & 1,533 & 1,064 \\
33 & 5 & 0,202 & 0,132 & 1,733 & 0,773 \\
33 & 1 & 0,180 & 0,088 & 1,633 & 0,489 \\
77 & 5 & 0,223 & 0,132 & 2,267 & 0,692 \\
77 & 1 &
\end{tabular}

Para os valores de Rz, pode-se observar também na Tabela 3 que o maior valor de rugosidade foi de 2,267 $\mu \mathrm{m}$ para o tamanho de grão de 39,9 $\mu \mathrm{m}$ e o menor valor foi $0,489 \mu \mathrm{m}$ para o tamanho de grão de 497 $\mu \mathrm{m}$. Os valores definidos na Tabela 3 correspondem aos valores médios de rugosidade para os parâmetros Ra e Rz. Nas Figuras de 4(a) a 4(d), são apresentados os acabamentos das superfícies exatamente após o processo de microusinagem para as condições definidas na Tabela 3. Usou-se um microscópio eletrônico de varredura marca Hitachi modelo TM3000 para realizar as análises. As fotos foram produzidas imediatamente após os testes de microusinagem. Entretanto, para a realização das análises de rugosidade, as amostras foram sopradas antes das medições com ar comprimido. Usou-se essa técnica para evitar a interferência no acabamento de sistemas de limpeza tradicionais como escovas ou pincéis.

Pode-se observar nas Figuras 4(a) e 4(c), com corpos de prova com tamanho de grão de $497 \mu \mathrm{m}$, que os riscos deixados pela ferramenta são mínimos e quase imperceptíveis, mostrando que o menor valor de avanço por dente empregado produziu um acabamento próximo do retificado. Esse fato pode ser confirmado em [12], onde no processo de retificação centerless o valor de acabamento de hastes cilíndricas no parâmetro Rz ficou entre 0,56 e $1,0 \mu \mathrm{m}$, sendo o valor de $1,2 \mu \mathrm{m}$ o valor máximo para o controle de qualidade de peças no chão de fábrica. Conforme pode ser observado na Tabela 3, a rugosidade no parâmetro Rz variou de 0,489 a $1,064 \mu \mathrm{m}$ em valores gerais.

Para as Figuras 4(b) e 4(d), também com corpos de prova de tamanho de grão de $497 \mu \mathrm{m}$, nota-se que os riscos deixados pela ferramenta são mais significativos para o valor de avanço por dente de $0,005 \mu \mathrm{m}$, onde se pode observar também que esse fato ocorreu independente da variação da velocidade de corte. Dessa forma, pode-se considerar que de acordo com observações visuais das Figuras 4(a) a 4(d) que, assim como ocorre no processo de fresamento em escala macro, o avanço por dente deixa marcas proporcionais ao deslocamento na peça usinada. Entretanto, foi realizado um planejamento fatorial para comprovar a influência de cada parâmetro do processo sobre as respostas Ra e Rz.

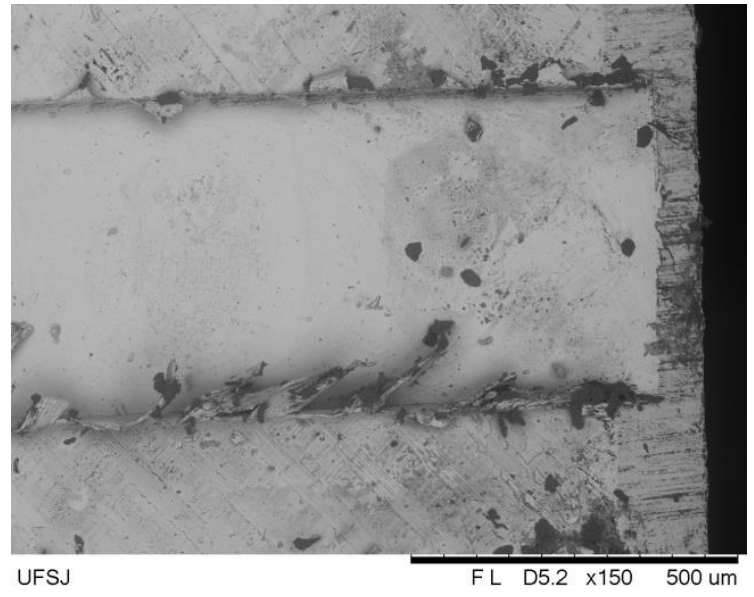

(a) $\mathrm{vc}=33 \mathrm{~m} / \mathrm{min}$ e $\mathrm{f}_{\mathrm{Z}}=0,001 \mathrm{~mm} / \mathrm{z}$

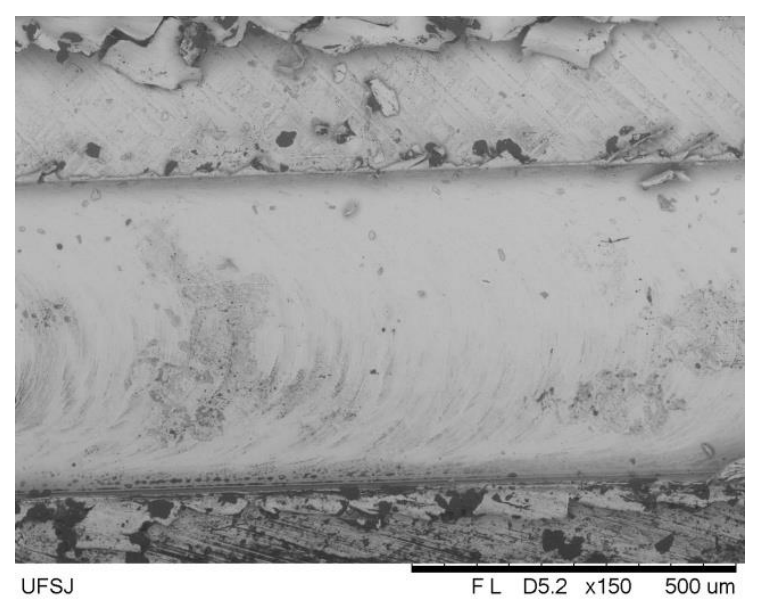

(b) $\mathrm{vc}=33 \mathrm{~m} / \mathrm{min}$ e $\mathrm{f}_{\mathrm{Z}}=0,005 \mathrm{~mm} / \mathrm{z}$ 


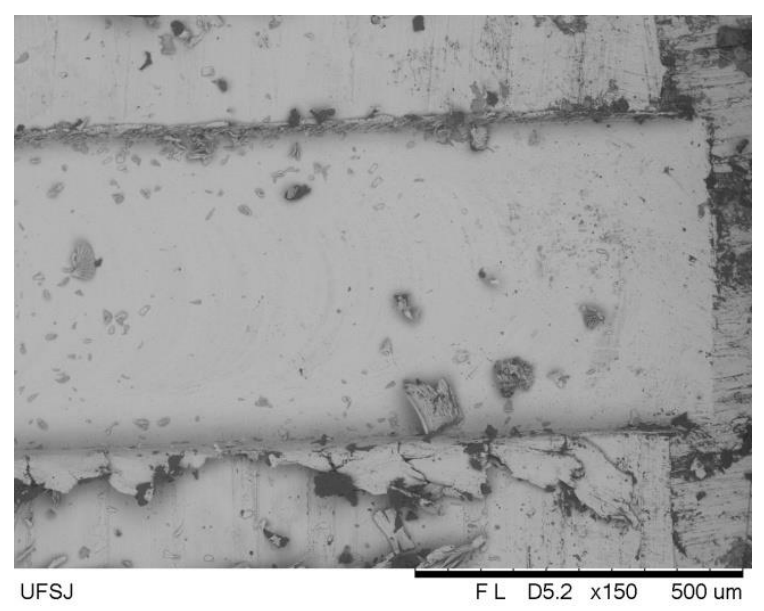

(c) $\mathrm{vc}=77 \mathrm{~m} / \mathrm{min}$ e $\mathrm{f}_{\mathrm{Z}}=0,001 \mathrm{~mm} / \mathrm{z}$

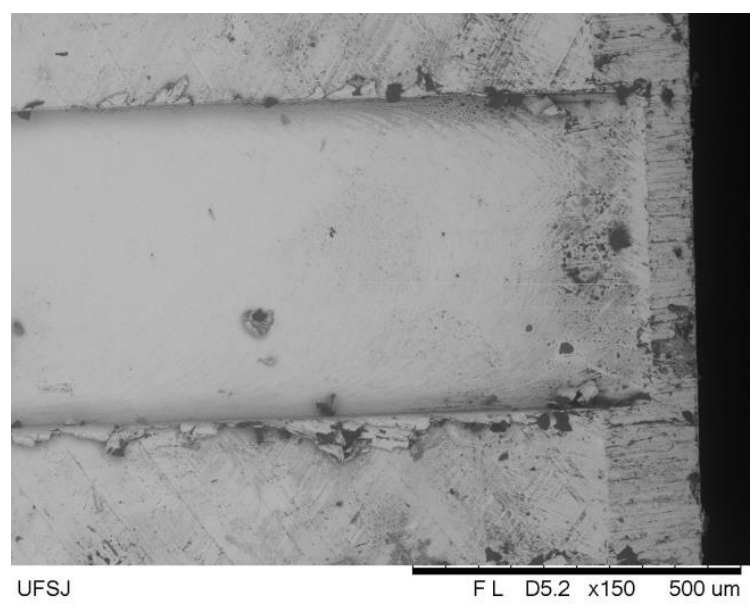

(d) $\mathrm{vc}=77 \mathrm{~m} / \mathrm{min}$ e $\mathrm{f}_{\mathrm{Z}}=0,005 \mathrm{~mm} / \mathrm{z}$

Figura 4: Detalhe das superfícies fresadas - tamanho de grão $497 \mu \mathrm{m}$

Nas Figuras de 5(a) a 5(d), são mostradas as superfícies fresadas para os corpos de prova com tamanho de grão de 39,9 $\mu \mathrm{m}$. Nota-se que os riscos deixados pela ferramenta são mais visíveis para os dois valores de avanço e de velocidade de corte empregados. Os riscos da ferramenta que correspondem ao deslocamento por dente, assim como ocorreu no fresamento dos corpos de prova com tamanho de grão de $497 \mu \mathrm{m}$, são maiores para o avanço por dente de 0,005 $\mu \mathrm{m}$. Entretanto, considerando-se o valor do avanço por dente de $0,001 \mu \mathrm{m}$ e a qualidade da superfície do corpo de prova, nota-se que o acabamento foi inferior ao produzido no corpo de prova com tamanho de grão de $497 \mu \mathrm{m}$ independente do valor de velocidade de corte utilizado. Dessa forma, observa-se que de modo geral o tamanho de grão do corpo de prova teve influência sobre a superfície fresada.

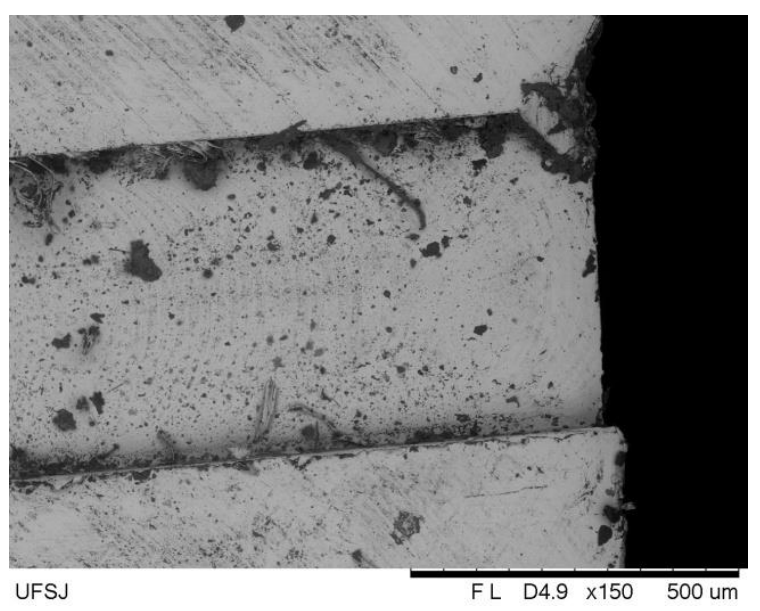

(a) $\mathrm{vc}=33 \mathrm{~m} / \mathrm{min}$ e $\mathrm{f}_{\mathrm{Z}}=0,001 \mathrm{~mm} / \mathrm{z}$

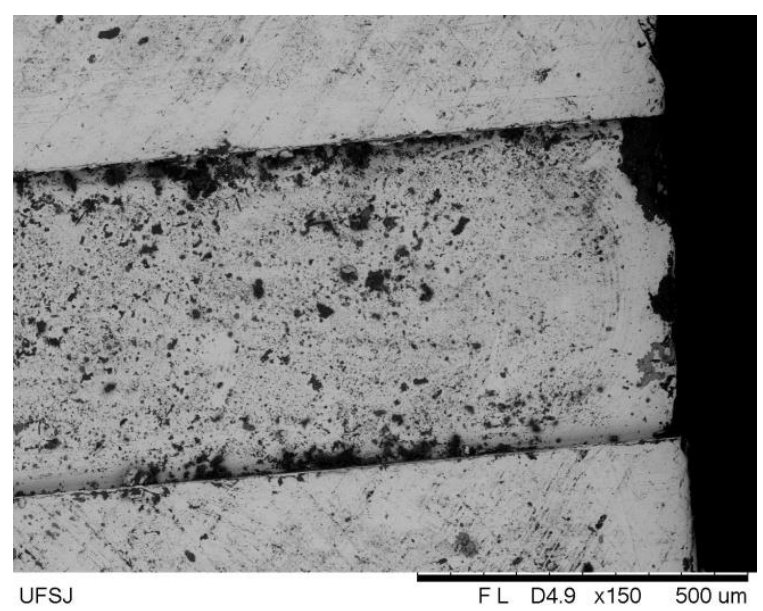

(b) $\mathrm{vc}=33 \mathrm{~m} / \mathrm{min}$ e $\mathrm{f}_{\mathrm{Z}}=0,005 \mathrm{~mm} / \mathrm{z}$ 


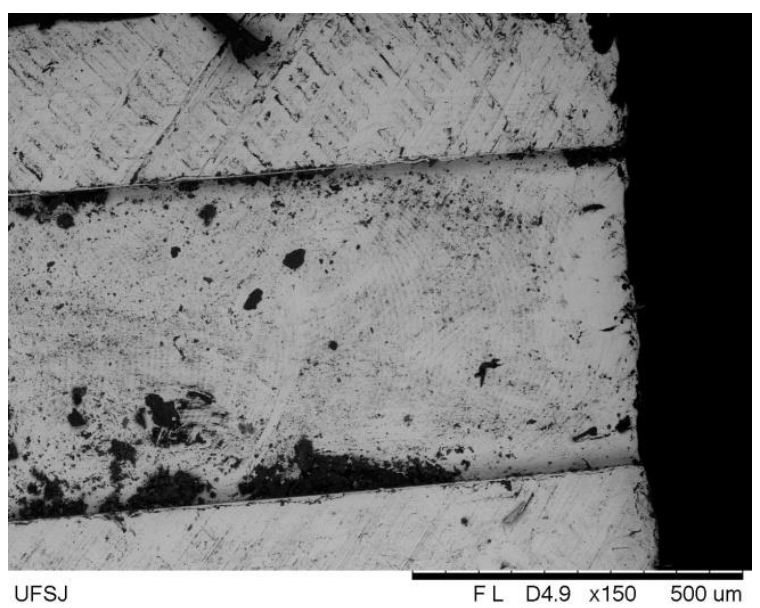

(a) $\mathrm{vc}=77 \mathrm{~m} / \mathrm{min}$ e $\mathrm{f}_{\mathrm{Z}}=0,001 \mathrm{~mm} / \mathrm{z}$

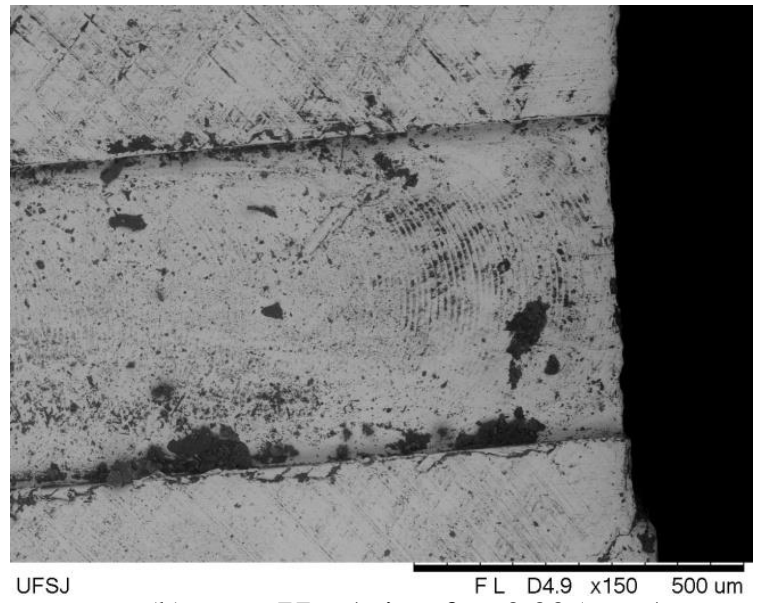

(b) $\mathrm{vc}=77 \mathrm{~m} / \mathrm{min}$ e $\mathrm{f}_{\mathrm{Z}}=0,005 \mathrm{~mm} / \mathrm{z}$

Figura 5: Detalhe das superfícies fresadas - tamanho de grão 39,9 $\mu \mathrm{m}$

A Tabela 4 apresenta o P-valor da análise de variância (ANOVA) para o tamanho de grão, avanço por dente e velocidade de corte. O P-valor indica quais os efeitos no sistema são estatisticamente significativos. Se o P-valor é menor ou igual a 0,05 , o efeito é considerado significativo. Um nível de $0,05 \%$ é o nível de significância que implica que existe $95 \%$ de probabilidade de o efeito ser significativo. $\mathrm{O}$ valor $\mathrm{R}^{2}$ ajustado mostrado na análise de variância indica quão bem o modelo prevê respostas para novas observações. Valores maiores para o $\mathrm{R}^{2}$ ajustado sugerem um modelo com maior capacidade preditiva [13]. De acordo com a Tabela 4, apenas o tamanho de grão teve influência sobre a resposta rugosidade, considerando os valores de Ra e Rz. Além disso, quando se observa o P-valor para os parâmetros de rugosidade Ra e Rz, nota-se que não houve interação entre os parâmetros avanço, velocidade de corte e tamanho de grão sobre as rugosidades analisadas. Pode-se inferir, a partir da Tabela 4, que a seção de corte pode mudar mediante variações do avanço por dente e também pelo tamanho de grão que está sendo usinado. Dessa forma, pode-se afirmar que dependendo do tamanho de grão que está sendo cortado, a qualidade superficial pode mudar significativamente.

Tabela 4: Análise de variância para as Rugosidades Ra e Rz

\begin{tabular}{lrrrr} 
& \multicolumn{1}{c}{ Fonte de Variação } & \multicolumn{2}{c}{ Ra $[\mu \mathrm{m}]$} & \multicolumn{2}{c}{$\mathrm{Rz}[\mu \mathrm{m}]$} \\
\cline { 2 - 5 } & F-valor & P-valor & F-valor & P-valor \\
\hline Tamanho do grão & 5,47 & $\underline{\mathbf{0 , 0 2 4}}$ & 12,47 & $\underline{\mathbf{0 , 0 0 1}}$ \\
Velocidade de Corte & 1,51 & 0,227 & 0,40 & 0,530 \\
Avanço por dente & 0,00 & 0,986 & 0,00 & 0,984 \\
Tamanho do grão $v s$. Velocidade de Corte & 0,35 & 0,556 & 0,62 & 0,437 \\
Tamanho do grão $v s$. Avanço por dente & 1,03 & 0,316 & 1,21 & 0,279 \\
Velocidade de Corte $v s$. Avanço por dente & 0,13 & 0,718 & 0,62 & 0,435 \\
Tamanho do grão $v s$. Veloc. de Corte $v s$. Avanço por dente & 0,37 & 0,548 & 0,00 & 0,960 \\
\multicolumn{2}{c}{$\mathrm{R}^{2}$ ajustado } & $70,65 \%$ & $74,71 \%$ \\
\hline
\end{tabular}

Pode-se observar no gráfico de efeitos principais das Figuras 6(a) e 6(b) que ocorre um decréscimo significativo das rugosidades nos parâmetros Ra e Rz quando ocorre uma variação do grão menor para o grão maior, ou seja, os corpos de prova com grãos maiores geraram valores de rugosidades Ra e Rz menores que os corpos de prova com grãos menores. Essa redução corresponde a uma redução de $50 \%$ do valor máximo de $0,18 \mu \mathrm{m}$ para um valor mínimo de $0,12 \mu \mathrm{m}$ considerando o parâmetro Ra conforme observado na figura 6(a). A mesma proporção foi observada na redução do parâmetro Rz que diminuiu de $1,79 \mu \mathrm{m}$ para $0,75 \mu \mathrm{m}$ correspondendo a $50 \%$, o que pode ser verificado na Figura 6(b). 
Observa-se também que, ao contrário do tamanho de grão, existe uma tendência ao aumento das rugosidades $\mathrm{Ra}$ e $\mathrm{Rz}$ influenciadas pelo avanço por dente. Porém, essa afirmação não pode ser verificada, pois de acordo com a Tabela $4 \mathrm{o}$ avanço por dente tem valores acima de 0,05 confirmando que não existe influência sobre as rugosidades Ra e Rz. Da mesma forma, considerando-se a variação da velocidade de corte, nota-se que não existe uma influência desse parâmetro de entrada sobre as respostas rugosidades Ra e $\mathrm{Rz}$, pois a velocidade de corte apresenta-se como uma reta constante dentro dos valores médios de rugosidade, de acordo com as Figuras 6(a) e 6(b). O P-valor para a velocidade de corte sobre as respostas rugosidade $\mathrm{Ra}$ e $\mathrm{Rz}$ foi de 0,227 e 0,530, respectivamente, estando muito acima do coeficiente de 0,05 de confiabilidade conforme observado na Tabela 4.

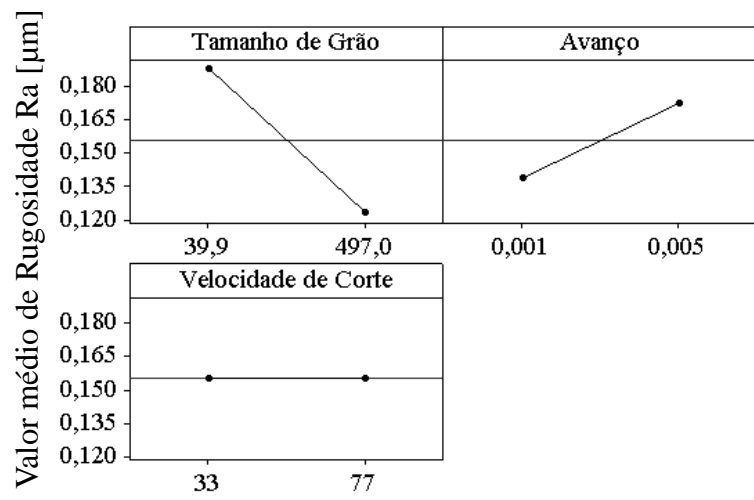

(a)

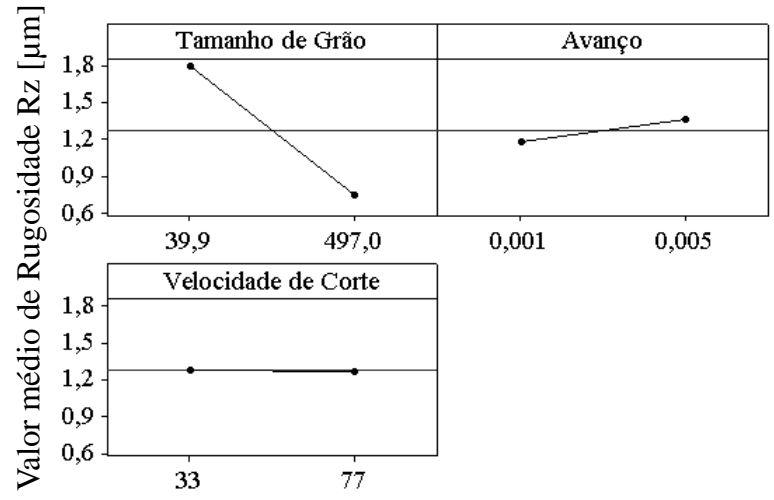

(b)

Figura 6: Gráficos de Efeitos principais (a) Rugosidade Ra e (b) Rugosidade Rz

Os gráficos de interação das Figuras 7(a) e 7(b) comprovam as informações da Tabela 4. Pode-se observar que não existe interação entre os parâmetros de entrada velocidade de corte, avanço por dente e tamanho de grão. De acordo com [13], existe uma interação entre os fatores quando a diferença na resposta entre os níveis de um fator não é a mesma em todos os níveis dos outros fatores. Dessa forma, pode ser observado nas Figuras 7(a) e 7(b) que a análise da rugosidade Ra e Rz nos níveis de baixo para alto $(0,001$ e $0,005 \mu \mathrm{m}$ para o avanço por dente e 33 e $77 \mathrm{~m} / \mathrm{min}$ para a velocidade de corte) mantém-se constante ou, em alguns casos, aumenta e/ou diminui nos níveis mais altos (avanço por dente de $0,005 \mu \mathrm{m}$ e velocidade de corte igual $77 \mathrm{~m} / \mathrm{min}$ ). Assim, pode-se afirmar que, como não existe uma inversão dos valores das respostas $\mathrm{Ra}$ e Rz gerando uma interceptação entre as retas, também não existe interação entre os parâmetros de entrada.

Interação (valores médios) Rugosidade Ra [ $\mu \mathrm{m}]$

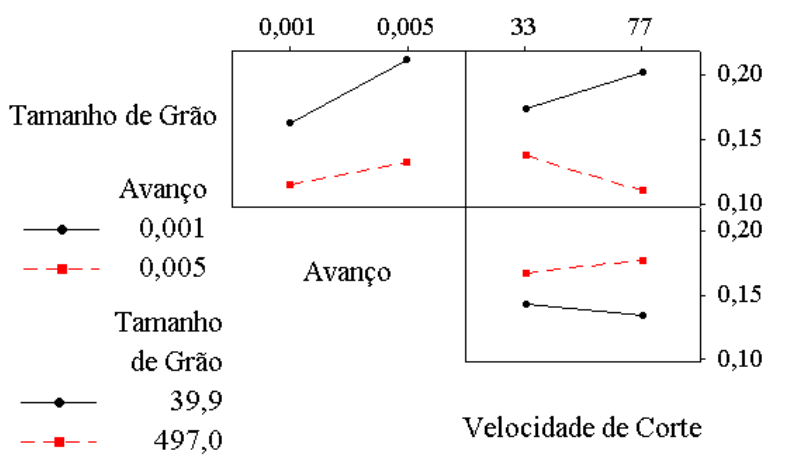

(a)
Interação (valores médios) Rugosidade Rz [ $\mu \mathrm{m}]$

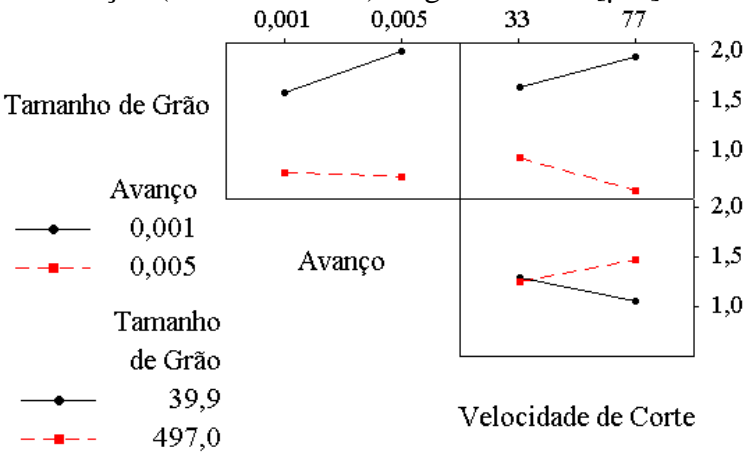

(b)

Figura 7: Interações: avanço, tamanho de grão e velocidade de corte (Rugosidade Ra e Rz)

Portanto, os efeitos da rugosidade $\mathrm{Ra}$ e Rz estão relacionados com mínima espessura do cavaco removido, sendo uma combinação dos efeitos do raio de ponta da ferramenta, microestrutura do material relacionada com o tamanho de grão. De acordo com [4], o avanço também tem influência no processo de microfresamento, porém neste trabalho não se pode confirmar essa influência do avanço por dente sobre a 
rugosidade. De acordo com os dados obtidos, pode-se definir que materiais com tamanhos de grão pequenos provocam o aumento das rugosidades Ra e Rz.

Deve-se salientar que nos experimentos foram usadas ferramentas com diâmetro de $0,5 \mathrm{~mm}$ que corresponde a aproximadamente 12,5 vezes o tamanho do grão de $39,9 \mu \mathrm{m}$ e em outros casos os grãos tinha aproximadamente o mesmo tamanho da ferramenta $(500 \mu \mathrm{m} \cong 497 \mu \mathrm{m})$. O raio de quina foi medido com uma ampliação de 10.000 vezes utilizando-se um MEV, sendo encontrado o valor de 2,77 $\mu \mathrm{m}$, conforme Figura 9. Portanto, pode-se afirmar que a influência do raio de quina da ferramenta em relação ao tamanho de grão de $39,9 \mu \mathrm{m}$ pode ser mais significativa que nos grãos com tamanho de $497 \mu \mathrm{m}$ que, neste caso, corrobora com as afirmações feitas por [4]. Porém, grãos menores podem apresentar uma recuperação elástica proporcional ao tamanho, mas que pode representar alterações na superfície, picos e vales, em quantidade maior por área, devido ao tamanho reduzido destes influenciando diretamente no micro formato da superfície usinada.

Dessa forma, como foi afirmado por [14, $\underline{15}]$, as rugosidades Ra e Rz não tiveram um aumento relativo com o aumento do avanço por dente. Assim, como estatisticamente esse parâmetro não teve influência sobre as rugosidades nos parâmetros $\mathrm{Ra}$ e $\mathrm{Rz}$ de acordo com a confiabilidade de $95 \%$, pode-se suportar que a recuperação elástica do material foi a maior responsável pela influência da variação do tamanho de grão, sendo significativa para a melhoria da rugosidade.

As Figuras 8 e 9 mostram a geometria das ferramentas empregadas nos testes de microfresamento. A Figura 8(a) mostra uma microfresa em uma vista frontal antes do início dos testes experimentais. Observa-se que a mesma apresenta uma geometria bem positiva com uma aresta de corte bem afiada. Essa geometria é considerada afiada, porém, segundo [16], o raio de quina da ferramenta é relativamente maior que a espessura do cavaco deformado e não consegue evitar em alguns casos deformações plásticas, microtrincas ou quebra das microfresas. Dessa forma, o modelo de corte preciso considerando uma ponta afiada ocorre ao longo da aresta da ferramenta arredondada. Esse efeito influi significativamente no ângulo de inclinação da aresta de corte que passa a ser negativo afetando os esforços de cisalhamento.

Ainda segundo [16], quando a espessura de corte é menor que a espessura do cavaco, não são mais gerados cavacos durante o processo de corte, ocorrendo uma deformação plástica do material. Esse fato pode contribuir na geração de uma rugosidade Ra e Rz com valores mais altos quando são empregados tamanhos de grão menores. De acordo com [17], é bem conhecido e dominado nos processos de usinagem que os materiais não homogêneos têm grande influência sobre a rugosidade do material usinado e sobre a força de corte em processos de microusinagem.

A Figura 8(b) mostra a mesma microfresa com um desgaste típico que ocorreu após um determinado tempo de usinagem. Para que fosse evitada a influência do desgaste das ferramentas nas respostas rugosidade $\mathrm{Ra}$ e Rz, foram empregadas seis fresas para cada tamanho de grão. Assim, para o corpo de prova com tamanho de grão de $39,9 \mu \mathrm{m}$, foram fresados 36 entalhes de $11 \mathrm{~mm}$, totalizando um comprimento usinado de $396 \mathrm{~mm}$ e um desgaste de $0,025 \mathrm{~mm}$ medido na região frontal da microfresa. Para o grão maior com $497 \mu \mathrm{m}$, foram fresados 24 entalhes de $11 \mathrm{~mm}$ totalizando um comprimento de $264 \mathrm{~mm}$ e um desgaste de $0,026 \mathrm{~mm}$.

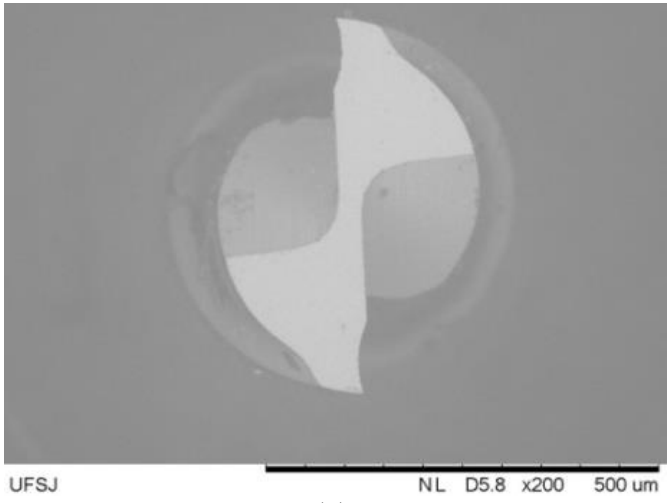

(a)

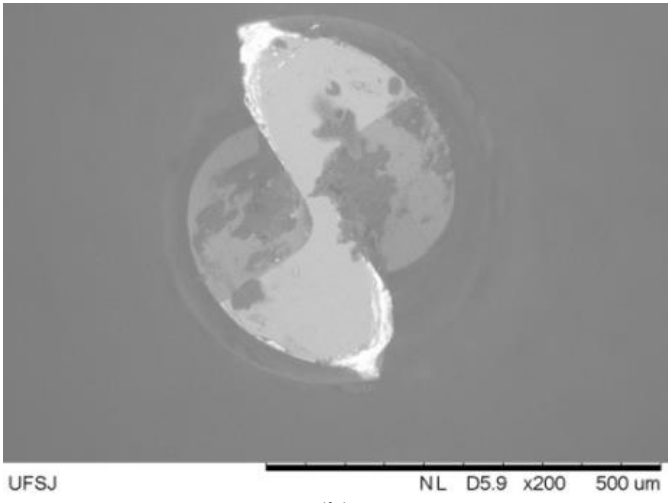

(b)

Figura 8: Microscopia eletrônica das microfresas; (a) ferramenta nova e (b) ferramenta desgastada 


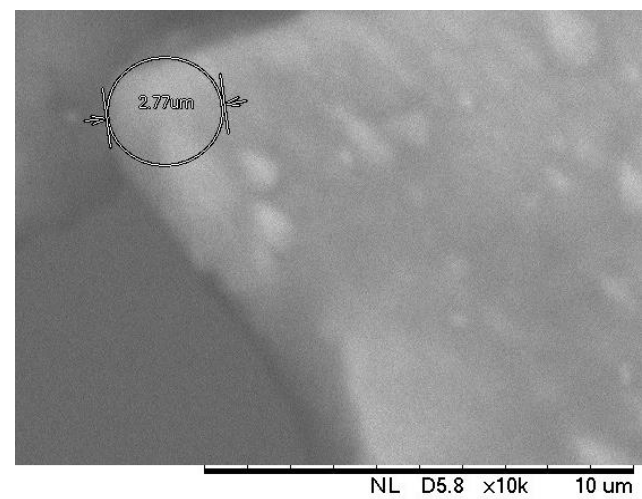

Figura 9: Microscopia eletrônica do raio de quina da microfresa

A Figura 10(a) mostra uma vista lateral da ferramenta desgastada, com ampliação de 100 vezes e a Figura 10(b) mostra uma ampliação de 250 vezes da mesma região. Pode-se notar que ocorreu um arredondamento da aresta de corte da ferramenta considerando o raio de quina, como pode ser observado na Figura 8(b) e também um arredondamento no raio de ponta, conforme a Figura 10(b). O raio de ponta $\mathrm{r}_{\varepsilon}$ é formado entre a aresta principal e a aresta secundária de corte, sendo que para a ferramenta nova esse valor corresponde a $20 \mu \mathrm{m}$ de acordo com catálogo do fabricante [18].

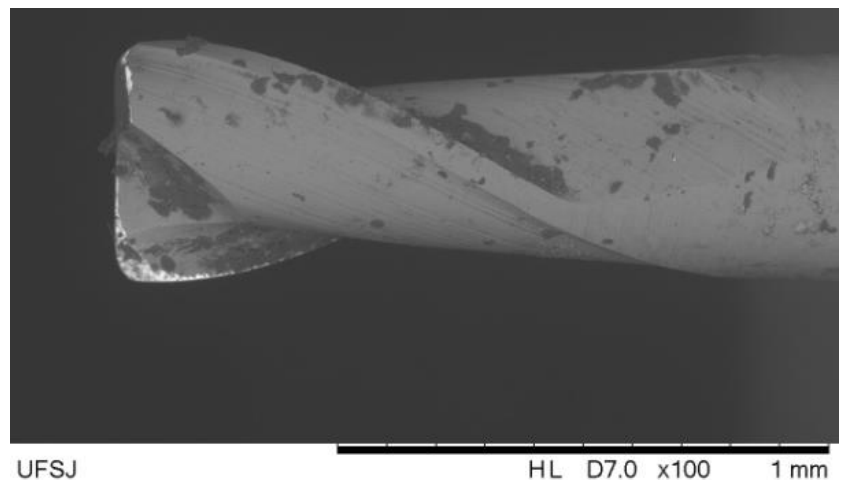

(a)

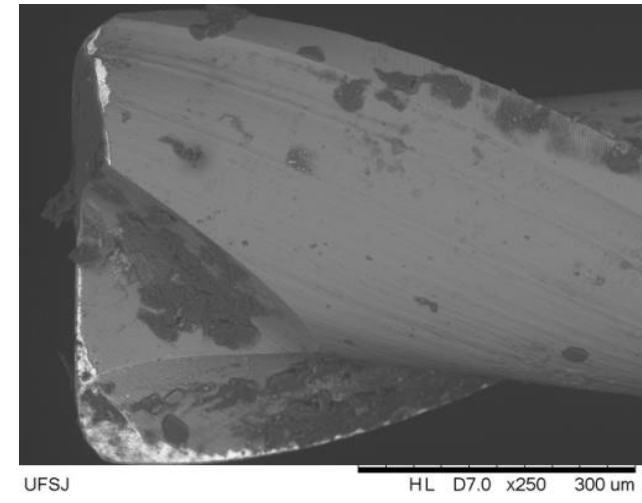

(b)

Figura 10: Microscopia eletrônica das microfresas; (a) ferramenta desgasta e (b) região desgastada

Observa-se na Figura 10(b), que ocorreu um desgaste na superfície de saída e um arredondamento no raio de ponta $r_{\varepsilon}$ após alguns testes experimentais, este valor de arredondamento na Figura 10(b) foi igual a 90 $\mu \mathrm{m}$ sendo o maior valor registrado durante os experimentos. Entretanto, a metodologia empregada foi usar seis ferramentas novas durante todos os experimentos de forma aleatória. Portanto, considerando o planejamento fatorial $2^{\mathrm{k}}$ e três repetições para cada condição experimental, foram realizados 24 experimentos, sendo que cada microfresa realizou o microfresamento de 4 canais.

Pode-se assim, inferir que a variabilidade dos valores de rugosidade Ra e Rz ocorreu em razão dos diferentes níveis de desgaste observados nas fresas. Paralelamente, no diâmetro máximo da microfresa ocorre o maior valor de velocidade de corte que provoca uma grande deformação nos cavacos. Em seguida, esta deformação inicial o cavaco sofre uma compressão significativa, pois o cavaco ainda não é separado e começa a formação do segmento seguinte. Na região do centro da ferramenta, a velocidade de corte é próxima de zero gerando um cisalhamento imperfeito do cavaco e um efeito de esmagamento que segundo [19], nos processos de fresamento, pode melhorar o acabamento da superfície usinada minimizando a rugosidade.

Além disso, o rugosímetro utilizado nas medições tem um sistema mecânico de medição com um apalpador de ponta cônica de $60^{\circ}$. A região de contato onde foi realizada a medição da rugosidade dentro dos entalhes correspondeu exatamente à linha de centro teórica do canal, conforme pode ser observado na Figura 11. Portanto, esta região está relacionada com o centro da microfresa que não apresentou desgaste de quina e nem desgaste no raio de ponta $r_{\varepsilon}$ como pode ser observado nas Figuras 8 (b) e 10(b). 


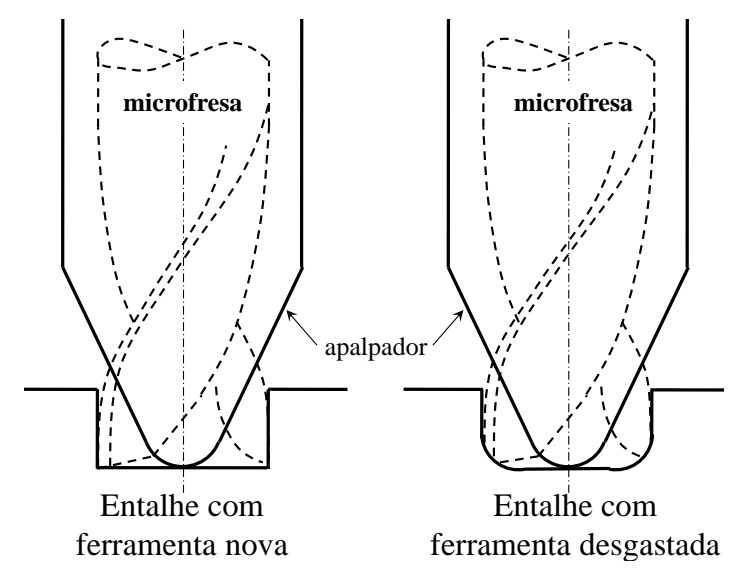

Figura 11: Detalhe das medições de rugosidade

\section{CONCLUSÕES}

Por meio dos resultados obtidos pelo processo de microfresamento em aço AISI H13 com diferentes tamanhos de grãos austeníticos, pode-se concluir que aços com grãos austeníticos maiores apresentam valores menores de rugosidade nos parâmetros $\mathrm{Ra}$ e Rz. O avanço por dente, assim como ocorre geralmente nos processos de macrofresamento não apresentou influência nos valores de rugosidade Ra e Rz. A variação da velocidade de corte também não gerou nenhuma alteração nos valores de rugosidade Ra e Rz. Além disso, observou-se que a interação das variáveis não demonstrou influência significativa sobre as respostas rugosidade Ra e Rz. A variação do comprimento de usinagem no processo de microfresamento de aços endurecidos foi de 264 a $396 \mathrm{~mm}$ com um desgaste máximo de 0,026 mm. Finalmente, conclui-se que a variação do tamanho de grão austenítico tem influência não apenas nas propriedades físicas dos materiais, mas, dependendo da escala de miniaturização dos componentes mecânicos, o tamanho de grãos poderá ter influência significativa durante o processo de microusinagem, principalmente na qualidade superficial.

\section{AGRADECIMENTOS}

Os autores gostariam de agradecer a SANDVIK Coromant pelo fornecimento do ferramental e a Gerdau Aços Finos Piratini pelo fornecimento do aço AISI H13.

\section{BIBLIOGRAFIA}

[1] LAURO C.H. Análise Numérica com Validação Experimental das Forças de Corte no Processo de Microfresamento de Aços Endurecidos com Variação do Tamanho de Grão Austenítico, Dissertação (Mestrado em Engenharia Mecânica), Universidade Federal de São João del-Rei. 83p., 2011.

[2] ARAMCHAROEN A., MATIVENGA P.T. "Size effect and tool geometry in micromilling of tool steel", Precision Engineering, v. 33, p. 402-407, 2009.

[3] WEULE H., HÜNTRUP V., TRITSCHLER H. "Micro-cutting of steel to meet new requirements in miniaturization", In: Annals of CIRP, v. 50, p. 61-64, 2001.

[4] VOGLER M. P., KAPOOR S. G., DEVOR R. E. "On the Modeling and Analysis of Machining Performance in Micro-End Milling, Part II: Cutting Force Prediction", ASME Journal of Manufacturing Science and Engineering, v. 126, p.685-694, 2004.

[5] CRISTOFARO, S. de, FUNARO, N., FERITI, G. C., et al.,. "High-speed micro-milling: Novel coatings for tool wear reduction International", Journal of Machine Tools \& Manufacture, v. 63, p. 16-20, 2012.

[6] CHANG, W., SUN, J., LUO, X., et al., "Investigation of micro structured milling tool for deferring tool wear", Wear, v. 271, n. 9-10, p. 2433-2437, 2011.

[7] SILVA, A.L.V.C., MEI P.R., Aços e ligas especiais. $3^{a}$ ed. Edição Revista e ampliada, Editora Edgard Blücher, São Paulo, 664p, 2010.

[8] CALLISTER JUNIOR W.D., Fundamentos da Ciência e Tecnologia dos Materiais - Uma abordagem Integrada, $2^{\mathrm{a}}$ ed., LTC - Livros Técnicos e Científicos Editora, 700p, 2006. 
[9] REED-HILL, R.E., ABBASCHIAN, R., ABBASCHIAN, L. Physical Metallurgy principles, 4th ed., Cengage Learning Editora, 749p, 2010.

[10] LEE, W.B., CHEUNG, C.F. "A dynamic surface topography model for the precision of nano-surface generation in ultra-precision machining", International Journal of Mechanical Sciences, v. 43, p. 961-991, 2001.

[11] ÇOLAKA, O., CAHIT, K. C., KAYACANA, M.C., "Milling surface roughness prediction using evolutionary programming methods", Materials \& Design, v. 28, n. 2, p. 657-666, 2007.

[12] RASCALHA, A., BRANDÃO, L.C., RIBEIRO FILHO, S.L.M. "Optimization of the dressing operation using load cells and the Taguchi method in the centerless grinding process", International Journal of Advanced Manufacturing Technology, v. 63, p.1-10, 2012.

[13] WERKEMA M. C. C., AGUIAR S., Planejamento e análise de experimentos: como identificar as principais variáveis influentes em um processo, Belo Horizonte, UFMG, 1996.

[14] BENARDOS P.G., VOSNIAKOS G. "Predicting surface roughness in machining: a review". International Journal of Machine Tools \& Manufacture, v. 43, p. 833-844, 2003.

[15] LIU, X., VOR, R.E. de, KAPOOR, S.G., et al., "The mechanics of machining at the microscale: assessment of the current state of the science", Transactions of ASME Journal Manufacturing Science Engineering, v. 166, p. 666-678, 2004.

[16] CHAE J., PARK S.S., FREIHEIT T., "Investigation of micro-cutting operations", International Journal of Machine Tools \& Manufacture, v. 46, p. 313-332, 2006.

[17] VOGLER M.P., DeVOR R.E., KAPOOR S.G., "Microstructure-level force prediction model for micromilling of multi-phase materials", Journal of Manufacturing Science and Engineering, v. 125, p. 202-209, 2003.

[18] SANDVIK, COROMANT, Catalogo principal C-2900:4 POR/01, 790p, 2012.

[19] SUTTER, G., LIST, G., "Very high speed cutting of Ti-6Al-4V titanium alloy - change in morphology and mechanism of chip formation", International Journal of Machine Tools \& Manufacture, v. 66, p. 37- 43, 2013. 